\title{
Involvement of neurotrophic signaling in doxorubicin-induced cardiotoxicity
}

\author{
DEHUA LIAO $^{1 *}$, CHEN ZHANG $^{2 *}$, NI LIU $^{1}$, LIZHI CAO $^{1}$, CHANGSHUI WANG $^{3}$, \\ QINGYAN FENG ${ }^{4}$, DUNWU YAO $^{1}$, MINGHUI LONG ${ }^{1}$ and PEI JIANG ${ }^{3}$ \\ ${ }^{1}$ Department of Pharmacy, Hunan Cancer Hospital, Changsha, Hunan 410013; ${ }^{2}$ Department of Cardiology, \\ ${ }^{3}$ Institute of Clinical Pharmacy and Pharmacology and ${ }^{4}$ Department of Neurology, Jining First People's Hospital, \\ Jining Medical University, Jining, Shandong 272000, P.R. China
}

Received May 24, 2019; Accepted October 8, 2019

DOI: $10.3892 /$ etm.2019.8276

\begin{abstract}
Dose dependent cardiotoxicity is the primary side effect of doxorubicin (DOX), but the underlying molecular mechanisms remain unclear. An increasing amount of evidence has demonstrated that neurotrophic signaling plays a pivotal role in both neurons and the heart, but the biological association between neurotrophic signaling and DOX-induced cardiotoxicity remains unknown. The present study determined the level of neurotrophins and their receptors in the heart of rats following DOX administration. DOX was administered 7 times at a dose of $2.5 \mathrm{mg} / \mathrm{kg}$ once every 2 days via intraperitoneal injection. The present study revealed that cardiac injury parameters, such as creatine kinase (CK), creatine kinase-myocardial bound, lactate dehydrogenase, troponin $\mathrm{T}$ and aspartate transaminase in serum were significantly increased in the DOX group. Both the gene and protein expression of brain-derived neurotrophic factor (BDNF) and nerve growth factor (NGF) in the heart were markedly decreased following DOX treatment. Notably, the protein level of BDNF in the serum was inhibited in DOX-treated rats, whereas DOX induced a significant increase in the protein level of NGF in the serum. DOX induced a significant decrease in the level of tropomyosin-associated kinase A (TrkA) and the ratio of pTrkA/TrkA and pTrkB/TrkB. Furthermore, the administration of DOX suppressed downstream protein kinase B and extracellular signal regulated kinase phosphorylation. The present study first demonstrated that BDNF/TrkB signaling and NGF/TrkA signaling were altered by DOX, which indicated that neurotrophic signaling was involved in DOX-induced cardiotoxicity.
\end{abstract}

Correspondence to: Professor Pei Jiang, Institute of Clinical Pharmacy and Pharmacology, Jining First People's Hospital, Jining Medical University, Jining, Shandong 272000, P.R. China

E-mail: jiangpeicsu@sina.com

*Contributed equally

Key words: doxorubicin, neurotrophic signaling, cardiotoxicity, brain-derived neurotrophic factor/TrkB, nerve growth factor/TrkA

\section{Introduction}

Doxorubicin (DOX) is effective in the treatment of hematological malignancies, solid tumors, soft tissue sarcomas and breast cancer $(1,2)$. However, adverse reactions such as neutropenia, heart arrhythmias, irreversible cardiomyopathy, congestive heart failure and neurotoxicity significantly limit its clinical use (3). Cardiotoxicity is the typical dose dependent adverse effect of DOX $(4,5)$, but the molecular mechanism underlying DOX-induced cardiotoxicity remains unclear.

Brain derived neurotrophic factor (BDNF) and nerve growth factor (NGF) are the typical neurotrophic factors (6), which play a pivotal role in neuronal function, development, survival and differentiation of neurons (7). It is well known that the tropomyosin-associated kinase (Trk) family include high-affinity receptors for these two neurotrophins. NGF and BDNF bind to TrkA and TrkB, respectively $(6,8)$. The activation of these receptors will always be accompanied by the stimulation of several intracellular signaling pathways, leading to different cellular responses and promoting survival, growth, differentiation and maintenance of both neuronal and non-neuronal cells. Although neurotrophins are necessary factors for neurons, neurotrophins and their receptors also play a pivotal role in non-neuronal cells. Skaper (9) has reported that the state of vascular smooth muscle cells and cardiomyocytes is regulated by NGF/TrkA signaling. Govoni et al (10) has reported that NGF is important in protecting against cardiac physiopathology. In addition, BDNF also exhibited a cardioprotective effect in the heart. Hang et al (11) reported that BDNF could effectively attenuate DOX-induced cardiac dysfunction through activating protein kinase B (Akt) signaling in rats. Zhao et al (12) also reported that 7,8-dihydroxyflavone $(7,8$-DHF) attenuated DOX-induced cardiotoxicity by regulating the BDNF/TrkB signaling pathway both in vivo and in vitro. Feng et al (13) reported that $\mathrm{BDNF} /$ TrkB signaling is necessary for normal heart function. The aforementioned evidence suggested that neurotrophins exert their nutritional effect in both the brain and heart $(11,14,15)$. A previous study demonstrated that a DOX-induced chemobrain was always accompanied by decreasing levels of neurogenesis, BDNF and TrkB (16). 
However, it is not yet known whether the neurotrophic signaling pathway in the heart is associated with DOX-induced cardiotoxicity. Although the role of the BDNF/TrkB/Akt pathway in DOX-induced cardiotoxicity have been reported in a previous study, none of these studies have reported the BDNF/TrkB and NGF/TrkA pathway in DOX-induced cardiotoxicity in the same time. Therefore, the present study aimed to investigate the roles of the BDNF/TrkB and NGF/TrkA signaling pathways in DOX-induced cardiotoxicity.

\section{Materials and methods}

Animals. Male Sprague-Dawley rats $(\mathrm{n}=18$; age, 8 weeks; weight, 200-230 g) were provided by the Experimental Animal Center of Hunan Cancer Hospital. All animals were kept under standard conditions with water and food readily available. All methods and experimental protocols in the present program were approved by the Animal Care and Use Committee of Hunan Cancer Hospital (protocol no. 016/2017). The present study conformed to the Guide for the Care and Use of Laboratory Animals (Chinese Council).

Experimental design. Animals were randomized and allotted to two groups (9 per group). Normal saline was administered to rats in the control group $(2 \mathrm{ml})$. By means of intraperitoneal injection, DOX was administered every 2 days at a dose of $2.5 \mathrm{mg} / \mathrm{kg}$ and a total of 7 injections were given to each rat in the DOX group. The dose and treatment duration was chosen based on previous research (17).

The rats were anesthetized with sodium pentobarbital $(50 \mathrm{mg} / \mathrm{kg})$ via intraperitoneal injection at day 14 of the experiment. Blood samples $(1.5 \mathrm{ml})$ were then collected directly from the left ventricle of the heart. Following the blood collection, the rats were sacrificed with an overdose sodium pentobarbital $(220 \mathrm{mg} / \mathrm{kg})$ and cardiac tissues dissected from the left ventricle were immediately removed from each rat. Physiological saline was used to wash the cardiac tissues. Western blotting and PCR were performed following cardiac tissue dissection. Histopathological examination was performed with the remaining cardiac tissues, which were fixed in $10 \%$ neutral-buffered formalin.

Serum biochemical analysis. The plasma was centrifuged at $2,000 \mathrm{x} \mathrm{g}$ for $10 \mathrm{~min}$ at $4^{\circ} \mathrm{C}$ and the supernatant was used for determination of cardiac injury parameters. Cardiac injury parameters, such as creatine kinase $(\mathrm{CK})$ activity, creatine kinase-myocardial bound (CK-MB) activity, troponin $\mathrm{T}$ activity and lactate dehydrogenase (LDH) activity in the serum, were determined using an automatic biochemical analyzer (ADVIA ${ }^{\circledR} 2400$, Siemens Ltd.). Furthermore, the clinical toxicity marker aspartate aminotransferase (AST) was also measured.

Histopathological examination. For the histological analysis, $10 \%$ neutral-buffered formalin was used to fix the hearts for $10 \mathrm{~min}$ at room temperature. The hearts were then embedded in paraffin and sliced into $5-\mu \mathrm{m}$ sections.

For hematoxylin and eosin (H\&E) staining, sections were deparaffinized using dimethyl benzene, dehydrated using alcohol for $5 \mathrm{~min}$ at room temperature, washed with distilled water and stained with hematoxylin at room temperature for $30 \mathrm{~min}$. After the removal of excess stain, the sections were incubated with $1 \%$ acid alcohol for $30 \mathrm{sec}$ at room temperature and dehydrated using an alcohol gradient (70, 85, 95 and $100 \%$; 5 min per condition) at room temperature. Sections were stained with $0.5 \%$ eosin at room temperature for $5 \mathrm{~min}$, incubated with $95 \%$ alcohol at room temperature for 2 min and with xylene at room temperature for $10 \mathrm{~min}$ and finally sealed with a neutral balsam. Sections were examined under the light microscope and photographs were taken.

For masson's trichrome staining, sections were stained with Weigert solution (Sigma-Aldrich; Merck KGaA) for 5-10 min. After that, sections were treated with Ponceau fuchsin acid solution for 5-10 min, immersed in 2\% acetic acid aqueous solution for $1 \mathrm{~min}$ and then differentiated in $1 \%$ phosphomolybdic acid aqueous solution for 3-5 min. Without washing with water, the sections were treated with aniline blue for 5 min then immersed in $0.2 \%$ acetic acid aqueous solution for $1 \mathrm{~min}$. Slices were permeabilized with xylene and mounted with neutral resin. Sections were examined under the light microscope and photographs were taken. For each rat five visual fields were randomly selected and used to calculate the average myocardial collagen volume. Assessments of the histology results were performed by a pathologist who was blinded to the treatment groups.

Reverse transcription-quantitative PCR (RT-qPCR) analysis. RT-qPCR analysis was performed in the present study in order to assess the expression levels of BDNF and NGF using the $2^{-\Delta \Delta \mathrm{Cq}}$ method of quantification, as previously reported (18). According to the manufacturer's protocol for the use of TRIzol ${ }^{\circledR}$ (Thermo Fisher Scientific, Inc.), total RNA was extracted from the isolated cardiomyocytes. cDNA was synthesized using The RevertAid RT reverse transcriptase kit (Thermo Fisher Scientific, Inc.) was used for RT at the conditions of $42^{\circ} \mathrm{C}$ for $15 \mathrm{~min}$ and $95^{\circ} \mathrm{C}$ for 3 min. A SYBR-Green PCR kit (Thermo Fisher Scientific, Inc.) and gene-specific primers were used to perform qPCR with a Bio-Rad Cx96 Detection System (Bio-Rad Laboratories, Inc.). cDNAs were amplified during 40 cycles with the primers presented in Table I. Thermocycling conditions were as follows: $50^{\circ} \mathrm{C}$ for $2 \mathrm{~min}, 95^{\circ} \mathrm{C}$ for $10 \mathrm{~min}$ and 40 cycles of $15 \mathrm{sec}$ at $95^{\circ} \mathrm{C}$, followed by an annealing/extension step for $1 \mathrm{~min}$ at $60^{\circ} \mathrm{C}$. Each reaction was repeated in triplicate and $\beta$-actin was used as an endogenous control.

Western blot analysis. Total protein was extracted from the cardiomyocytes using RIPA lysis buffer (200 mg tissue/1 ml buffer; cat. no. R0010; Beijing Solarbio Science \& Technology Co., Ltd.) and quantified using the Bradford method. Proteins were separated via electrophoresis and transferred to a polyvinylidene fluoride membrane. Samples were loaded on precast $12 \%$ SDS-PAGE gels with $\sim 50 \mu \mathrm{g}$ protein in each lane. The membranes were blocked for $1 \mathrm{~h}$ in TBST (25 mM Tris, $\mathrm{pH} 7.5$, $150 \mathrm{mM} \mathrm{NaCl}, 0.05 \%$ Tween-20) and 5\% non-fat dry milk. Blots were incubated at $4{ }^{\circ} \mathrm{C}$ overnight with antibodies against BDNF (Abcam; cat. no. ab1083191; 1:2,000); NGF (Abcam; cat. no. ab6199; 1:1,000); TrkA (Abcam; cat. no. ab76291; 1:3,000); p-TrkA (Tyr49; Cell Signaling Technology, Inc.; cat. no. 9141; 1:1,000); TrkB (Santa Cruz Biotechnology, Inc.; cat. no. sc377218; 1:200); p-TrkB (Tyr705; Thermo Fisher Scientific, 
Table I. List of primer sequences used in the PCR analysis.

\begin{tabular}{lllc}
\hline Gene & \multicolumn{1}{c}{ Sense primer (5-3') } & \multicolumn{1}{c}{ Antisense primer (5-3') } & Amplicon length (bp) \\
\hline BDNF & ATCCCATGGGTTACACGAAGGAAG & AGTAAGGGCCCGAACATACGATTG & 97 \\
NGF & ACATCAAGGGCAAGGAGG & GTGAGTCGTGGTGCAGTATG & 164 \\
$\beta$-actin & CATCCTGCGTCTGGACCTGG & TAATGTCACGCACGATTTCC & 116 \\
\hline
\end{tabular}

NGF, nerve growth factor; BDNF, brain derived neurotrophic factor.

Inc.; cat. no. PA538077; 1:500); p75NTR (ProteinTech Group, Inc.; cat. no. 55014-1-AP; 1:1,000); Akt (ProteinTech Group, Inc.; cat. no. 10176-2-AP; 1:500); pAkt (Ser473; Cell Signaling Technology, Inc.; cat. no. 4060; 1:3,000); Erk (Santa Cruz Biotechnology, Inc.; cat. no. sc514302; 1:200); pErk (Thr202/Tyr204; Cell Signaling Technology, Inc.; cat. no. 4695; $1: 2,000)$ and $\beta$-actin (ProteinTech Group, Inc.; cat. no. 66009-1-Ig; 1:4,000). Following several washes, the membranes were then incubated with the corresponding horseradish peroxidase-conjugated secondary antibodies, including goat anti-rabbit (1:2,000, cat. no. 7074P2) and horse anti-mouse (1:2,000, cat. no. 7076P2; both obtained from Cell Signaling Technology, Inc.). The bands were then detected using a chemiluminescence detecting system (ECL Western Blotting Substrate; cat. no. PE0010-B; Beijing Solarbio Science \& Technology Co., Ltd.). $\beta$-actin was used as an internal standard for all gels. The intensity of protein signals was quantified with Image $\mathrm{Lab}^{\mathrm{TM}}$ software (version 5.2.1; Bio-Rad Laboratories, Inc.).

Determination of serum BDNF and NGF levels. ELISA kits were purchased from Wuhan USCN Business Co., Ltd. and were used to determine plasma levels of BDNF (cat. no. SEA011Ra) and NGF (cat.no. SEA105Ra) levels, following the manufacturer's recommendations All experiments were performed in duplicate.

Statistical analysis. In the present study, all values are presented as the mean \pm standard deviation. Data analysis was performed using SPSS software (version 13.0; SPSS Inc.). A Student's t-test was used to make comparisons between two groups. Each experiment was repeated three times. A two-tailed $\mathrm{P}<0.05$ was considered to indicate a statistically significant difference.

\section{Results}

Effects of DOX on cardiotoxicity. The dosage of DOX chosen in the present study was equal or higher than a previously reported toxic dose (17). Therefore, it is enough to cause cardiotoxicity with the current dosage. In addition, the biochemical analysis results in the present study also showed that the DOX-induced cardiotoxicity model was successfully produced. The HE staining assay and Masson's trichrome staining were performed for the histopathological examination of heart tissues. As presented in Fig. 1A, the structure and order of the myocardial fibers was normal in the control group. On the contrary, disorganization of myofibrillar arrays and cytoplasmic vacuolization, as indicated by the arrows, were observed in DOX-treated rats, which indicated severe cardiac damage. Compared with the control group, a large number of blue collagen fibers were observed in the areas surrounding the myocardial fibers in DOX treated rats and extended to the myocardial interstitium in Masson's trichrome staining (Fig. 1B). In the H\&E staining, the percentage of vacuolated areas in the total myocardium tissue was significantly increased in DOX treated rats compared with the control $(\mathrm{P}<0.01$; Fig. $1 \mathrm{C})$. In addition, the result of the Masson's trichrome staining revealed that the collagen volume fraction was significantly increased in DOX treated rats $(\mathrm{P}<0.01$; Fig. 1D).

As the main serum biochemical marker of myocardial damage, the levels of cardiac enzymes (LDH, CK, CK-MB, AST and troponin T) in serum were evaluated following DOX treatment in the present study. As presented in Fig. 1E, when compared with the control group, the serum level of CK-MB was significantly increased in DOX treated rats $(\mathrm{P}<0.01)$. As presented in Fig. 1F, the serum CK level was significantly elevated following the administration of DOX CK $(\mathrm{P}<0.01)$. In comparison with the rats in the control group, DOX induced a significant increase in serum levels of LDH in the DOX group $(\mathrm{P}<0.05$; Fig. 1G). In addition, serum levels of AST $(\mathrm{P}<0.01$; Fig. $1 \mathrm{H})$ and troponin $\mathrm{T}(\mathrm{P}<0.01$; Fig. 1I) were also significantly elevated following the administration of DOX. Based on the aforementioned results, it can be concluded that DOX successfully induced myocardial injury.

Effects of DOX on the expression of BDNF and NGF. In the present study, the expression of BDNF and NGF in the serum and heart were evaluated. Compared with the control group, the level of BDNF in the serum was significantly decreased in DOX treated rats $(\mathrm{P}<0.01$; Fig. $2 \mathrm{~A})$, while the serum NGF concentration was significantly increased in the DOX group ( $\mathrm{P}<0.05$; Fig. 2D). As presented in Fig. 2B and $\mathrm{E}$, in comparison with the control group, significant decreases in the gene expression levels of BDNF $(\mathrm{P}<0.01)$ and NGF $(\mathrm{P}<0.01)$ were observed in the DOX group. Consistent with the gene expression, the protein levels of BDNF and NGF were also significantly decreased in the DOX group $(\mathrm{P}<0.01$; Fig. $2 \mathrm{C}$, and $\mathrm{P}<0.05$; Fig. $2 \mathrm{~F}$, respectively).

Effects of DOX on the expression of neurotrophic receptors. The expression level of neurotrophic receptors was determined using western blot analyses in the present study. The results revealed that the expression of the TrkB protein was not markedly decreased in the DOX group when compared with the control rats (Fig. 3B), whereas the pTrkB/TrkB ratio was significantly decreased in the DOX treated group $(\mathrm{P}<0.01$; Fig. 3C). The level of the TrkA protein (P<0.05; Fig. 3D) and 

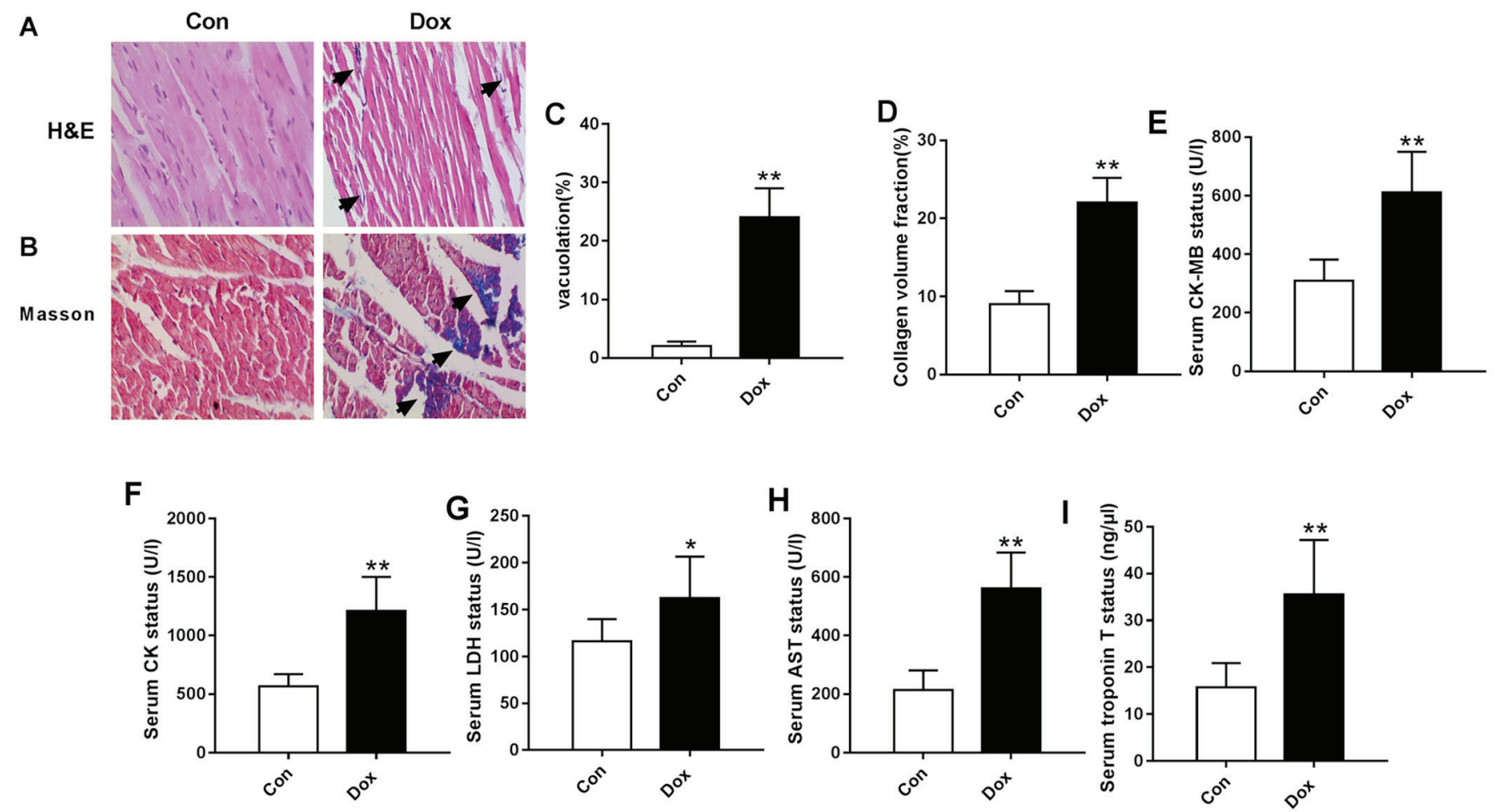

Figure 1. Effects of DOX on histopathological changes and myocardial damage biochemical markers. Magnification, x400. (A) H\&E staining of different groups. Arrows indicate disorganization of myofibrillar arrays and cytoplasmic vacuolization. (B) Masson staining of different groups. Arrows indicate a large number of blue collagen fibers. (C) The percentage vacuolated area of the total myocardium tissue from normal treated rats or DOX treated rats. (D) The collagen volume fraction was the proportion of the total area containing collagen. Serum level of (E) CK-MB, (F) CK, (G) LDH, (H) AST and (I) troponin T. Data are expressed as the mean \pm standard deviation $(\mathrm{n}=8-9)$. $\mathrm{P}<0.05$ and ${ }^{* *} \mathrm{P}<0.01$ compared with Con. DOX, doxorubicin; LDH, lactate dehydrogenase; AST, aspartate transaminase; H\&E, hematoxylin and eosin; CK-MB, creatine kinase-myocardial bound; Con, control.

the pTrkA/TrkA ratio $(\mathrm{P}<0.01$; Fig. $3 \mathrm{E})$ were all significantly decreased in the DOX-treated rats. p75NTR is another class of cell surface receptor, the expression of which was also evaluated in the present study. As presented in Fig. 3F, the expression level of p75NTR in the heart was not significantly changed following the administration of DOX.

Effects of DOX on the activation of Akt and Erk. It is well known that Akt and Erk play a pivotal role in the downstream signaling of various neurotropic factors, including BDNF (19). The phosphorylation of Akt and Erk indicated that these signaling proteins were activated. In the present study, the ratio of $\mathrm{pAkt} / \mathrm{Akt}(\mathrm{P}<0.01$; Fig. 4B) and pErk/Erk ( $<<0.05$; Fig. $4 \mathrm{C})$ were all significantly decreased following administration of DOX.

\section{Discussion}

DOX-induced cardiotoxicity triggers the development of cardiac dysfunction and congestive heart failure $(20,21)$, which limits its clinical application. The investigations in the present study demonstrated the potential role of neurotrophins in DOX-induced cardiotoxicity and the potential underlying molecular mechanisms have been elucidated. The major findings of the present study highlight several new and notable points: i) Protein and gene expression levels of BDNF and NGF in the heart were all decreased in DOX treated rats; ii) the ratio of $\mathrm{pTrkB} / \mathrm{TrkB}$ and $\mathrm{pTrkA} / \mathrm{Trk} \mathrm{A}$ were all decreased in the DOX group; iii) activation of downstream Akt and Erk signaling was compromised by DOX treatment.
The target organ for DOX is the heart and DOX-induced oxidative stress seems to be a leading cause of cardiomyopathy (22). As serum cardiac injury markers, the expression of LDH, CK and CK-MB were all increased in DOX-overdosed mice (3). Kobayashi et al (21) previously demonstrated that these biochemical parameters are important indicators of cardiac injury in DOX-induced cardiotoxicity. The results of the present study demonstrated that the level of CK, CK-MB, LDH and AST were all increased in the DOX-induced cardiac injury model, which indicated that DOX induced severe cardiotoxicity. The leakage of AST, CK, troponin T and LDH in the blood stream was always caused by damage to the myocardial cell membrane (23). The HE staining of disorganization of myofibrillar arrays, cytoplasmic vacuolization, myofibrillar degeneration, myocyte disruption and fibrosis revealed DOX-induced cardiotoxicity. With the Masson's staining, a typical pathological manifestation of myocardial remodeling was also observed following administration of DOX, that is, a large number of blue collagen fibers were observed in the areas surrounding the myocardial fibers (24).

As classical neurotrophins, more evidence has suggested that BDNF and NGF play a significant role in cardiovascular development and pathophysiology $(10,15)$. Previous studies have indicated that tissue NGF level has been recognized as a biomarker of heart disease and that the protective effects of NGF in the heart were based on pleiotropic action and the development of angiogenesis $(10,25)$. BDNF is expressed in tissues such as the heart, atherosclerotic vessels and vascular smooth muscle cells (26). In addition, patients with heart 
A

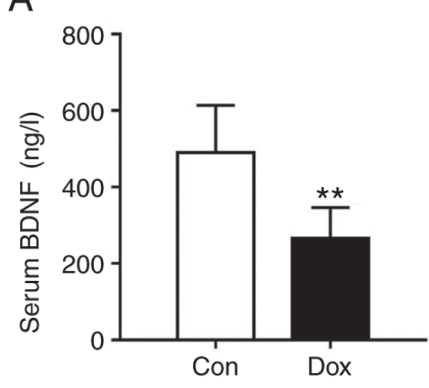

D

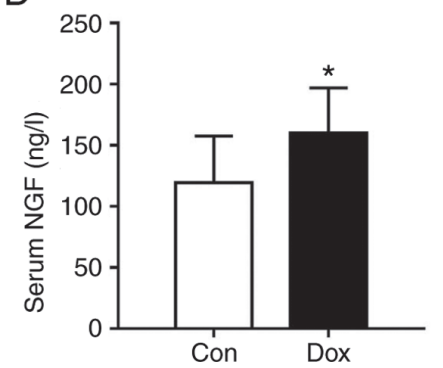

B

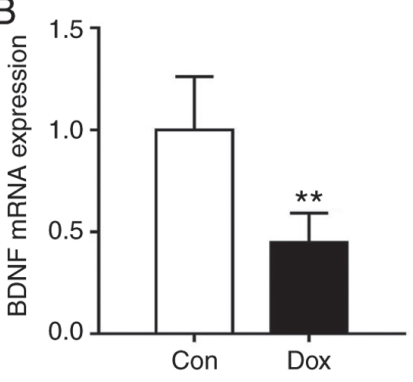

E

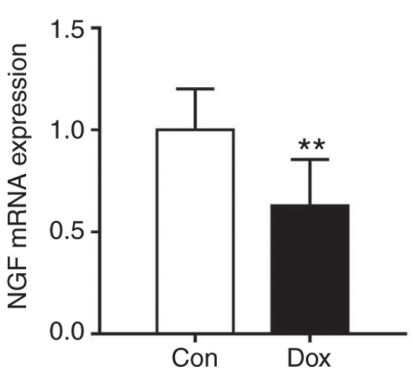

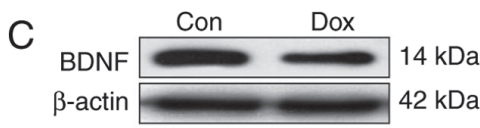

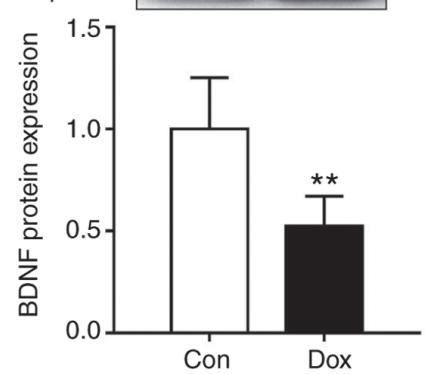

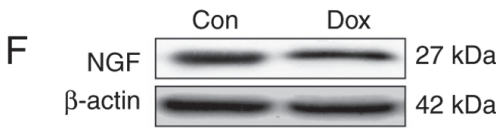

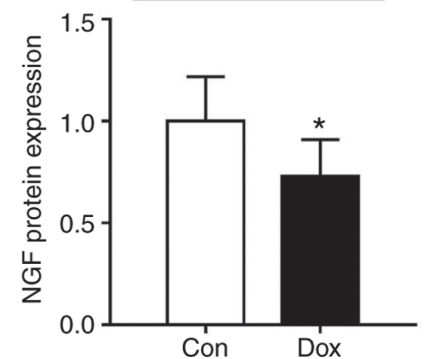

Figure 2. Effects of DOX on the expression of BDNF and NGF in the serum and heart. (A) BDNF concentration in the serum. (B) BDNF mRNA levels in the heart. (C) BDNF protein expression levels in the heart. (D) NGF concentration in the serum. (E) NGF mRNA levels in the heart. (F) NGF protein levels in the heart. Data are expressed as the mean \pm standard deviation $(n=8-9)$. ${ }^{*}<0.05$ and ${ }^{* *} \mathrm{P}<0.01$ compared with Con. NGF, nerve growth factor; BDNF, brain derived neurotrophic factor; DOX, doxorubicin; Con, control.
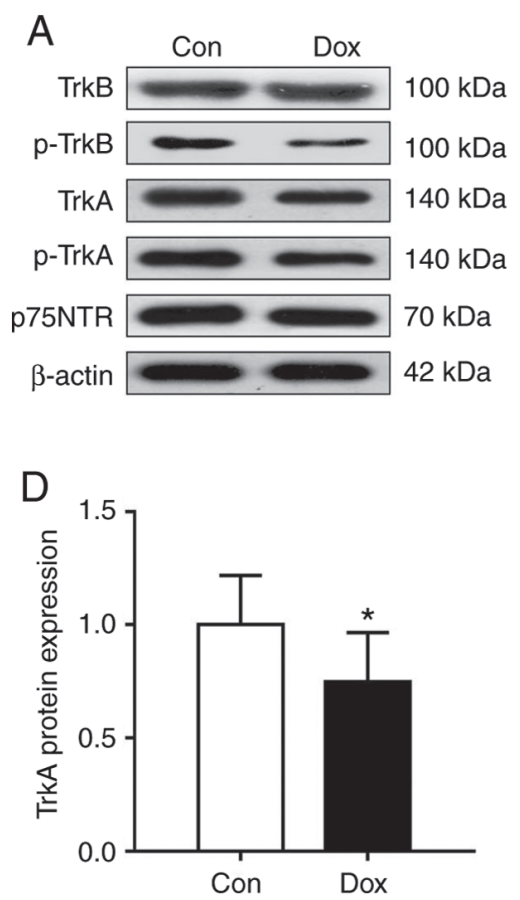
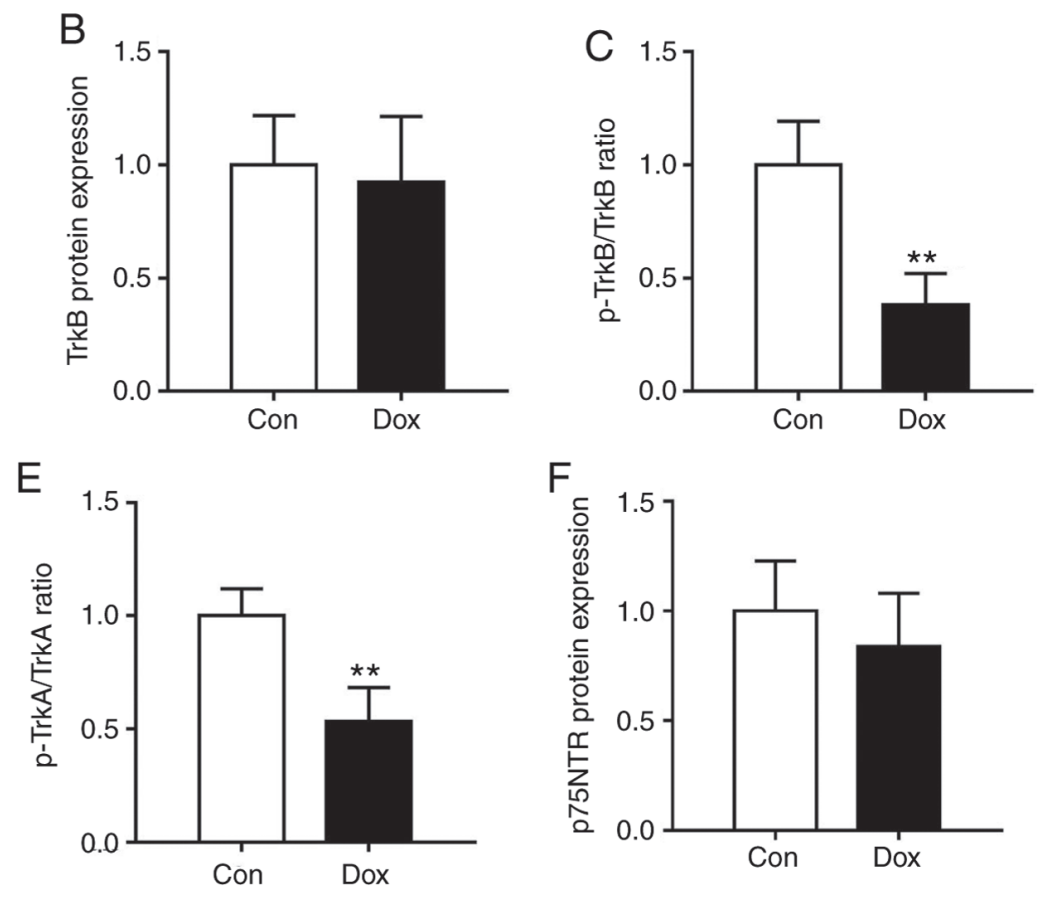

Figure 3. Effects of DOX on the protein levels of neurotrophic receptors in the heart. (A) Representative western blots. (B) TrkB expression levels. (C) p-TrkB/TrkB ratio. (D) TrkA expression levels. (E) p-TrkA/TrkA levels (F) p75NTR. Data are expressed as the mean \pm standard deviation ( $=8-9)$. ${ }^{*} \mathrm{P}<0.05$ and ${ }^{* *} \mathrm{P}<0.01$ compared with Con. Trk, tropomyosin-associated kinase; Dox, doxorubicin; Con, control; p, phosphorylated.

failure always exhibited a significant decrease in plasma BDNF levels (27). In agreement with the previous finding, the protein level of serum BDNF was suppressed in the DOX treated group in the present study. However, serum NGF protein levels were increased in the DOX-induced injury model. It was speculated that a compensatory mechanism was the foremost 
A
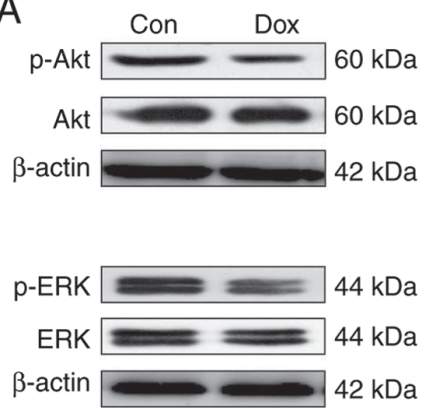
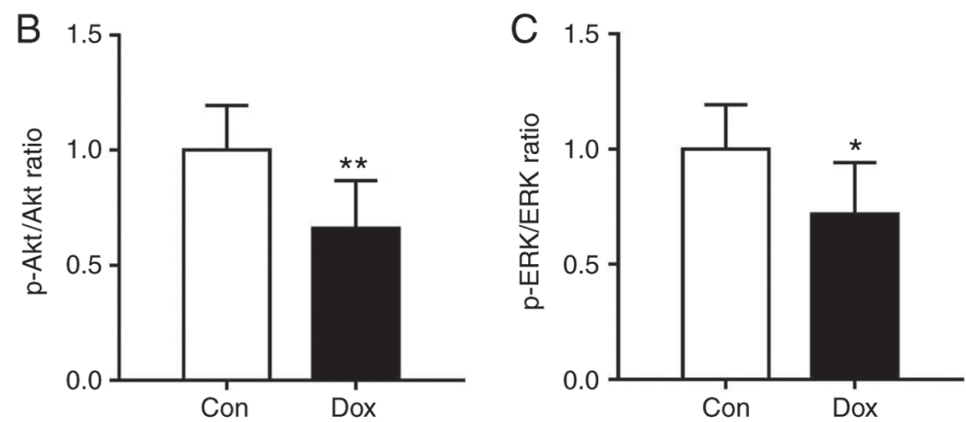

Figure 4. Effects of DOX on the activation of Akt and Erk. (A) Representative western blots. (B) pAkt/Akt ratio. (C) pErk/Erk ratio. Data are expressed as the mean \pm standard deviation $(\mathrm{n}=8-9)$. ${ }^{*} \mathrm{P}<0.05$ and ${ }^{* * *} \mathrm{P}<0.01$ compared with Con. ERK, extracellular signal regulated kinase; Akt, protein kinase $\mathrm{B}$; $\mathrm{p}$, phosphorylated; DOX, doxorubicin; Con, control.

reason for the enhancement of NGF. DOX induced a decrease in the protein and mRNA expression of BDNF and NGF in the present study. The protein expression levels of BDNF and NGF were consistent with the gene expression levels in the heart of DOX treated rats. However, the protein expression of NGF in the serum was not positively associated with gene expression of NGF in the present study. The results were in accordance with those of previous studies. Hang et al (11) reported that the protein level of BDNF was decreased in DOX-treated rat ventricles and the administration of BDNF could effectively upregulate BDNF and its receptor TrkB. The study results of Zhao et al (12) revealed that small-molecule TrkB agonist 7,8-DHF could attenuate DOX-induced cardiotoxicity by activating Akt. Kaye et al (28) have also reported that the expression and release of NGF from the heart were modified following pathological examination.

The 'shared' p75NTR and high-affinity Trk are two different classes of cell surface receptors, which mediate the biological activity of BDNF, NGF and p75NTR $(29,30)$. Previous studies have demonstrated that intact BDNF/TrkB and NGF/TrkA signaling play an important role in enhancing normal cardiac calcium cycling and normal cardiovascular system function $(13,31)$. After the association between NGF and the TrkA receptor was demonstrated, it was revealed that this triggers TrkA receptor autophosphorylation, which is also true for BDNF/TrkB signaling. Therefore, the protein level of TrkA, TrkB, p75NTR and phosphorylation of Trk were all investigated in the present study. The TrkA protein level was decreased in DOX treated rats. Both the ratio of $\mathrm{p}-$ TrkA/TrkA and $\mathrm{p}-\mathrm{TrkB} / \operatorname{TrkB}$ were decreased in the DOX group. The results from the present study also indicated that DOX suppressed activation of the downstream Akt and Erk pathway, which was detected by the phosphorylation of its mediators Erk and Akt, respectively. This result was in accordance with previous findings (32). Hang et al (11) also demonstrated that BDNF/TrkB signaling is necessary in preventing cardiotoxicity following administration of DOX. In addition, the beneficial effect of BDNF was abolished by BDNF scavenger Fc-fused TrkB (TrkB-Fc) or Akt inhibitor, which revealed the potent protective role of BDNF against Dox-induced cardiotoxicity by activating Akt signaling (11). Thus, as BDNF/TrkB and NGF/TrkA signaling was necessary for the function of neurotrophins in both neurons and heart, it was speculated that neurotrophic signaling may be a key factor of DOX-induced cardiotoxicity.
It is a limitation of the present study that direct evidence was not provided to verify the functions of these two pathways in attenuating or deteriorating the toxicity of DOX to the heart. To provide the direct evidence supporting BDNF/TrkB and NGF/TrkA signaling pathways in attenuating DOX induced cardiotoxicity a cell line-based assay to show that upregulation of BDNF and NGF can protect the cells from DOX-induced cardiotoxicity in heart cells is warranted in future studies.

To the best of our knowledge, the present study is the first to demonstrate that the signaling pathway of BDNF/TrkB and NGF/TrkA, and the downstream Akt and Erk pathway in the heart could be altered following administration of DOX in rats, which raises the possibility for the involvement of neurotrophic signaling in DOX-induced cardiotoxicity. Taken together, the results from the present study lead to an improved understanding of the role of neurotrophic signaling in DOX-induced cardiotoxicity and provided evidence for new strategies to prevent and decrease chemotherapy-induced cardiotoxicity.

\section{Acknowledgements}

Not applicable.

\section{Funding}

The present study was supported by the National Natural Science Foundation of China (grant no. NSFC:81603206), the Project of Hunan Provincial Science \& Technology Department (grant no. 2019JJ80093) and Health and Family Planning Commission Foundation of Hunan Province (grant no. C2019064).

\section{Availability of data and materials}

The datasets used and/or analyzed during the present study are available from the corresponding author upon reasonable request.

\section{Authors' contributions}

PJ, QF and DL designed the study and wrote the protocol. DL, CZ, NL and LC performed the experiments and analyzed the data. DY, ML and CW established the animal models and 
collected the data. DL and CZ drafted the manuscript. PJ and DL revised the manuscript content. All authors read and approved the final manuscript.

\section{Ethics approval and consent to participate}

All methods and experimental protocols in the present program were approved by the Animal Care and Use Committee of Hunan Cancer Hospital (protocol no. 016/2017).

\section{Patient consent for publication}

Not applicable.

\section{Competing interests}

The authors declare that they have no competing interests.

\section{References}

1. Injac R, Perse M, Obermajer N, Djordjevic-Milic V, Prijatelj M, Djordjevic A, Cerar A and Strukelj B: Potential hepatoprotective effects of fullerenol $\mathrm{C} 60(\mathrm{OH}) 24$ in doxorubicin-induced hepatotoxicity in rats with mammary carcinomas. Biomaterials 29 : 3451-3460, 2008.

2. Hajra S, Patra AR, Basu A and Bhattacharya S: Prevention of doxorubicin (DOX)-induced genotoxicity and cardiotoxicity: Effect of plant derived small molecule indole-3-carbinol (I3C) on oxidative stress and inflammation. Biomed Pharmacother 101: 228-243, 2018

3. Abdel-Daim MM, Kilany OE, Khalifa HA and Ahmed AAM: Allicin ameliorates doxorubicin-induced cardiotoxicity in rats via suppression of oxidative stress, inflammation and apoptosis Cancer Chemother Pharmacol 80: 745-753, 2017.

4. Singal PK, Li T, Kumar D, Danelisen I and Iliskovic N Adriamycin-induced heart failure: Mechanism and modulation Mol Cell Biochem 207: 77-86, 2000.

5. Wang XY, Yang CT, Zheng DD, Mo LQ, Lan AP, Yang ZL, Hu F, Chen PX, Liao XX and Feng JQ: Hydrogen sulfide protects H9c2 cells against doxorubicin-induced cardiotoxicity through inhibition of endoplasmic reticulum stress. Mol Cell Biochem 363 419-426, 2011

6. Carito V, Ceccanti M, Ferraguti G, Coccurello R, Ciafrè S, Tirassa $\mathrm{P}$ and Fiore M: NGF and BDNF alterations by prenatal alcohol exposure. Curr Neuropharmacol 17: 308-317, 2019.

7. Martinez-Cengotitabengoa M, MacDowell KS, Alberich S, Diaz FJ, Garcia-Bueno B, Rodriguez-Jimenez R, Bioque M, Berrocoso E, Parellada M, Lobo A, et al: BDNF and NGF signalling in early phases of psychosis: Relationship with inflammation and response to antipsychotics after 1 year. Schizophr Bull 42: 142-151, 2016.

8. Snider WD: Functions of the neurotrophins during nervous system development: What the knockouts are teaching us. Cell 77: 627-638, 1994.

9. Skaper SD: Nerve growth factor: A neuroimmune crosstalk mediator for all seasons. Immunology 151: 1-15, 2017.

10. Govoni S, Pascale A, Amadio M, Calvillo L, D'Elia E, Cereda C, Fantucci P, Ceroni M and Vanoli E: NGF and heart: Is there a role in heart disease? Pharmacol Res 63: 266-277, 2011

11. Hang P,Zhao J, Sun L, Li M, Han Y, Du Z and Li Y: Brain-derived neurotrophic factor attenuates doxorubicin-induced cardiac dysfunction through activating Akt signalling in rats. J Cell Mol Med 21: 685-696, 2017.

12. Zhao J, Du J, Pan Y, Chen T, Zhao L, Zhu Y, Chen Y, Zheng Y, Liu Y, Sun L, et al: Activation of cardiac TrkB receptor by its small molecular agonist 7,8-dihydroxyflavone inhibits doxorubicin-induced cardiotoxicity via enhancing mitochondrial oxidative phosphorylation. Free Radic Biol Med 130: 557-567, 2019.

13. Feng N,Huke S, Zhu G, Tocchetti CG, Shi S, Aiba T, Kaludercic N, Hoover DB, Beck SE, Mankowski JL, et al: Constitutive $\mathrm{BDNF} /$ TrkB signaling is required for normal cardiac contraction and relaxation. Proc Natl Acad Sci USA 112: 1880-1885, 2015.
14. Zeng N, Xu J, Yao W, Li S, Ruan W and Xiao F: Brain-derived neurotrophic factor attenuates septic myocardial dysfunction via eNOS/NO pathway in rats. Oxid Med Cell Longev 2017: 1721434, 2017.

15. Pius-Sadowska E and Machalinski B: BDNF-A key player in cardiovascular system. J Mol Cell Cardiol 110: 54-60, 2017.

16. Park HS, Kim CJ, Kwak HB, No MH, Heo JW and Kim TW: Physical exercise prevents cognitive impairment by enhancing hippocampal neuroplasticity and mitochondrial function in doxorubicin-induced chemobrain. Neuropharmacology 133: 451-461, 2018.

17. Liao D, Xiang D, Dang R, Xu P, Wang J, Han W, Fu Y, Yao D, Cao L and Jiang P: Neuroprotective effects of dl-3-n-Butylphthalide against doxorubicin-induced neuroinflammation, oxidative stress, endoplasmic reticulum stress, and behavioral changes. Oxid Med Cell Longev 2018: 9125601, 2018.

18. Livak KJ and Schmittgen TD: Analysis of relative gene expression data using real-time quantitative PCR and the 2(-Delta Delta C(T)) method. Methods 25: 402-408, 2001

19. Alboni S, Tascedda F, Corsini D, Benatti C, Caggia F, Capone G, Barden N, Blom JM and Brunello N: Stress induces altered CRE/CREB pathway activity and BDNF expression in the hippocampus of glucocorticoid receptor-impaired mice. Neuropharmacology 60: 1337-1346, 2011.

20. Li K, Sung RY, Huang WZ, Yang M, Pong NH, Lee SM, Chan WY, Zhao H, To MY, Fok TF, et al: Thrombopoietin protects against in vitro and in vivo cardiotoxicity induced by doxorubicin. Circulation 113: 2211-2220, 2006.

21. Kobayashi M, Usui F, Karasawa T, Kawashima A, Kimura H, Mizushina Y, Shirasuna K, Mizukami H, Kasahara T, Hasebe N and Takahashi M: NLRP3 deficiency reduces macrophage interleukin-10 production and enhances the susceptibility to doxorubicin-induced cardiotoxicity. Sci Rep 6: 26489, 2016

22. Berthiaume JM and Wallace KB: Adriamycin-induced oxidative mitochondrial cardiotoxicity. Cell Biol Toxicol 23: 15-25, 2006.

23. Khan G, Haque SE, Anwer T, Ahsan MN, Safhi MM and Alam MF: Cardioprotective effect of green tea extract on doxorubicin-induced cardiotoxicity in rats. Acta Pol Pharm 71: 861-868, 2014.

24. Wu X, Qi X, Lu Y, Lin C, Yuan Y, Zhu Q, Yin Q, Li W, Li Y and Bian H: Liguzinediol protects against cardiac fibrosis in rats in vivo and in vitro. Biomed Pharmacother 80: 260-267, 2016.

25. Meloni M, Caporali A, Graiani G, Lagrasta C, Katare R, Van Linthout S, Spillmann F, Campesi I, Madeddu P, Quaini F and Emanueli C: Nerve growth factor promotes cardiac repair following myocardial infarction. Circ Res 106: 1275-1284, 2010.

26. Ejiri J, Inoue N, Kobayashi S, Shiraki R, Otsui K, Honjo T, Takahashi M, Ohashi Y, Ichikawa S, Terashima M, et al: Possible role of brain-derived neurotrophic factor in the pathogenesis of coronary artery disease. Circulation 112: 2114-2120, 2005.

27. Takashio S, Sugiyama S, Yamamuro M, Takahama H, Hayashi T, Sugano Y, Izumiya Y, Hokimoto S, Minamino N, Yasuda S, et al: Significance of low plasma levels of brain-derived neurotrophic factor in patients with heart failure. Am J Cardiol 116: 243-249, 2015.

28. Kaye DM, Vaddadi G, Gruskin SL, Du XJ and Esler MD: Reduced myocardial nerve growth factor expression in human and experimental heart failure. Circ Res 86: E80-E84, 2000.

29. Bassili M, Birman E, Schor NF and Saragovi HU: Differential roles of Trk and p75 neurotrophin receptors in tumorigenesis and chemoresistance ex vivo and in vivo. Cancer Chemother Pharmacol 65: 1047-1056, 2010.

30. Carito V, Venditti A, Bianco A, Ceccanti M, Serrilli AM, Chaldakov G, Tarani L, De Nicolò S and Fiore M: Effects of olive leaf polyphenols on male mouse brain NGF, BDNF and their receptors TrkA, TrkB and p75. Nat Prod Res 28: 1970-1984, 2014.

31. Hou Y, Jia L, Zhang Y, Ji W and Li H: Activation of the NGF/TrkA signaling pathway attenuates diabetic erectile dysfunction. Oncotarget 8: 105692-105702, 2017.

32. Liao D, Guo Y, Xiang D, Dang R, Xu P, Cai H, Cao L and Jiang P: Dysregulation of Neuregulin-1/ErbB signaling in the hippocampus of rats after administration of doxorubicin. Drug Des Devel Ther 12: 231-239, 2018. 